\title{
18 Fazit
}

Dieses Buch könnte mit einem abschließenden, instruktiven Beispiel eines Primärtextes enden, an dem sich die zentralen Erkenntnisse der Studie noch einmal exemplarisch aufzeigen lassen. Auf ein solches Beispiel wird allerdings bewusst verzichtet. Grund hierfür ist, dass die vorliegende Arbeit gerade über die primär exemplarische oder zumindest genregebundene Betrachtungsweise der Kontrafaktik, wie sie in der bisherigen Forschung dominiert, hinausgehen möchte. Zwar könnte man problemlos diesen oder jenen dystopischen, alternativgeschichtlichen oder satirischen Text als Beispiel fiktionalen, kontrafaktischen Erzählens anführen (und zahlreiche solcher Beispiele wurden im Verlauf der Arbeit auch diskutiert). Folgt man jedoch dem in dieser Studie vorgeschlagenen Analysemodell, so kann es den einen, paradigmatischen Fall der Kontrafaktik nicht geben, ebenso wenig wie den einen, paradigmatischen Fall realistischen oder fantastischen Erzählens. Kontrafaktik bezeichnet weder eine objektiv bestimmbare Texteigenschaft noch auch ein bestimmtes Genre, sondern eine basale Möglichkeit der Korrelierung von realer und fiktionaler Welt, welche sich in unterschiedlichen Genres, Medien und Texten auf ganz unterschiedliche Weise manifestieren kann.

Anstatt die theoretischen Überlegungen der Arbeit durch eine letztlich beliebige Exemplifikation auf problematische Weise zu vereindeutigen, werden abschließend - gleichsam in Form einer nachgereichten Werbeschrift für das in dieser Arbeit verfolgte Forschungsprojekt - noch einmal die zentralen theoretischen Ergebnisse zusammengetragen. Die folgende Auflistung verzichtet dabei bewusst auf eine narrative Rahmung, sondern versteht sich als Reihe erweiterter Stichpunkte, die einen schnellen Überblick über die wichtigsten Erkenntnisse der vorliegenden Studie ermöglichen soll.

Theoretisches Kernanliegen der Arbeit war es, die Analyse des kontrafaktischen Erzählens in der Kunst fiktionstheoretisch zu grundieren. Der neu eingeführte Begriff ,Kontrafaktik' bezeichnet signifikante Variationen realweltlichen Faktenmaterials innerhalb fiktionaler Medien. Kontrafaktik bildet also eine bestimmte Referenzstruktur respektive ein bestimmtes Erzählverfahren, das den Leser zu einer Aktualisierung ebendieser Referenzstruktur veranlasst. Während weite Teile der bestehenden literaturwissenschaftlichen Forschung zum fiktionalen kontrafaktischen Erzählen theoretischen Rückhalt bei anderen Wissenschaftsdisziplinen suchen - insbesondere bei der Geschichtswissenschaft - und sich zur Bestimmung des Erzählphänomens auf tendenziell kunstferne Kategorien beziehen - wie etwa die Plausibilität imaginierter historischer Entwicklung oder die Deckung kontrafaktischer Alternativen durch reale Quellen -, wurde in dieser Studie der Versuch unternommen, die Eigenständigkeit der Kontrafaktik 
als genuin künstlerisches Phänomen herauszustellen. Zu diesem Zweck wurden der spezifische Fiktionsstatus, die Bedingungen sowie die Funktionen kontrafaktischen Erzählens in künstlerischen Medien fokussiert; ferner wurde ein literaturwissenschaftliches Bestimmungs- und Beschreibungsinstrumentarium für das fiktionale kontrafaktische Erzählen entwickelt.

Eine fiktionstheoretische und verfahrensanalytische Sichtweise auf die Kontrafaktik bringt gegenüber bestehenden Forschungsansätzen eine Reihe von Vorteilen mit sich. Aufgelöst wird damit die in der Forschung weithin dominierende, letztlich aber rein konventionelle und fiktionstheoretisch unhaltbare, ausschließliche Kopplung der Kontrafaktik an das historische Erzählen, also die Einschränkung einer Betrachtung des fiktionalen kontrafaktischen Erzählens auf den Genrebereich der Alternate History. (Im Kontext des faktualen kontrafaktischen Denkens hat es eine solche Themen- oder Disziplinenbindung ohnehin nie gegeben; hier wird seit jeher davon ausgegangen, dass sich kontrafaktische Argumentationsformen auf ganz unterschiedliche Aspekte des Weltwissens beziehen können.) Betrachtet man das kontrafaktische Erzählen in der Kunst nicht als eine Genrevariante des historischen Erzählens, sondern als das, was es rein dem Begriff nach ist - nämlich als eine vermittelnde Abweichung von vorderhand nicht näher bestimmtem Faktenmaterial -, so wird der Blick dafür frei, dass kontrafaktische Referenzstrukturen nicht allein in Werken des Alternate History-Genres vorkommen, sondern auch eine zentrale Rolle in Genres wie Dystopie und Utopie, dem kreativen Dokumentarismus, der Autofiktion, der Satire oder dem Schlüsselroman spielen.

Ein fiktionstheoretischer Zugriff auf die Kontrafaktik ermöglicht es, über eine genremäßige Globalklassifikation von Texten hinauszugehen und sich stattdessen konkreten Referenzstrukturen sowie ihrer Bedeutung für das jeweilige Werk zuzuwenden. Damit werden in der Folge auch die Möglichkeiten eines Vergleichs von Werken ganz unterschiedlicher Genrezugehörigkeit sowie einer verfahrensanalytisch präzisen Untersuchung von Genrehybriden eröffnet.

Eine fiktionstheoretische Betrachtung der Kontrafaktik erlaubt es ferner, die hohe Kontextsensitivität des Erzählphänomens zu würdigen und diese Kontexte für die Interpretation der jeweiligen Werke produktiv zu machen. Der konkrete Interpretationsprozess - respektive die erfolgreiche Aktualisierung kontrafaktischer Referenzstrukturen - ist stets in hohem Grade abhängig von bestimmten Kontextfaktoren, insbesondere vom Weltwissen der jeweiligen Rezipienten, welches wiederum durch eine Vielzahl von Meta-Faktoren etwa Nationalität, Sprach- und Generationenzugehörigkeit - mitkonfiguriert wird. Kontrafaktische Texte verhalten sich gewissermaßen epistemisch parasitär zu den Fakten der realen Welt, die sie variieren. Das bedeutet im Umkehrschluss aber auch, dass kontrafaktische Interpretationen viel über das 
epistemische (und häufig auch moralisch-normative) ,Weltbild“ einer bestimmten Rezeptionsgemeinschaft aussagen. Besonders deutlich wird diese Abhängigkeit der Kontrafaktik von der jeweiligen epistemischen Situation in Fällen, bei denen eine Kenntnis der deutungsrelevanten Fakten nicht (mehr) als selbstverständlich vorausgesetzt werden kann, etwa deshalb, weil das betreffende Weltwissen nicht hinreichend konventionalisiert oder bereit historisch ,verwittert' ist, oder aber in Fällen, bei denen der Status der realweltlichen Fakten als Fakten umstritten ist. Im letztgenannten Fall können sich, wie am Beispiel von Christian Krachts Werk gezeigt wurde, Grenzfälle zwischen kontrafaktischem, unzuverlässigem und postmodern-metafiktionalem Erzählen ergeben.

Der für die Literaturwissenschaft vielleicht größte Vorteil eines referenzstrukturell-verfahrensanalytischen Zugriffs auf die Kontrafaktik besteht in seiner hohen Anschlussfähigkeit an Fragen der Textinterpretation und Hermeneutik, eine Anschlussfähigkeit, wie sie bei den bisher in der Forschung dominierenden Genre- und Klassifikationsmodellen nicht gegeben war. Die Abweichung von realweltlichem Faktenmaterial ist zwar eine notwendige, aber noch keine hinreichende Bedingung für die Designation eines Elements innerhalb einer fiktionalen Welt als kontrafaktisch. Zusätzlich muss diese Abweichung respektive Variation auch als signifikant eingeschätzt werden, sich also für die Interpretation des jeweiligen Werkes als deutungsrelevant erweisen. Es handelt sich bei der Identifikation kontrafaktischer Elemente mithin niemals um eine mechanistische Klassifikation oder um ein bloßes ,Auszählen“ positiver Texteigenschaften, sondern um eine hochgradig kontextsensitive Zuschreibung, die vom Leser im Rahmen eines dynamischen Interpretationsprozesses geleistet werden muss. Die in dieser Studie vorgelegten Textdeutungen können dabei als exemplarische Belege dafür dienen, auf welch vielfältige Weise kontrafaktische Referenzstrukturen in fiktionale Texte integrierbar sind und wie interpretatorisch reizvoll gerade auch jene Fälle sein können, bei denen das Vorliegen kontrafaktischer Elemente selbst zweifelhaft ist: etwa weil die Kontrafaktik in Richtung eines postmodernen Wahrheitszweifels ausfasert (Kracht), weil Variationen von Faktenmaterial sich primär auf die formale Dimension der Faktenpräsentation beziehen (Röggla) oder weil plausible Werkdeutungen auch ohne Einbeziehung kontrafaktischer Elemente möglich sind, diese Deutungen sich aber in normativ-politischer Hinsicht sehr deutlich von - nicht weniger plausiblen - kontrafaktischen Deutungen desselben Werks unterscheiden (Randt).

Als Ordnungsraster des Verhältnisses zwischen der als real designierten Welt und etwaigen fiktionalen Welten wurde eine Unterteilung in vier sogenannte real-fiktionale Weltvergleichsverhältnisse vorgenommen, die auf einer Kreuzklassifikation der Kategorien Realitätsübereinstimmung und Realitätsab- 
weichung sowie allgemeiner und konkreter Realitätsbezug (oder auch Faktenbezug) beruht. Eine allgemeine Übereinstimmung zwischen realer und fiktionaler Welt wurde als ,Realistik‘, eine konkrete Übereinstimmung zwischen beiden als ,Faktik` bezeichnet; demgegenüber wurde eine allgemeine Abweichung zwischen fiktionaler und realer Welt als ,Fantastik‘ und eine konkrete Abweichung als ,Kontrafaktik' bezeichnet. Interpretatorisch relevant ist diese Aufteilung deshalb, weil unterschiedliche Weltvergleichsverhältnisse - immer verstanden als Interpretationsentscheidungen durch den jeweiligen Leser - mit unterschiedlichen Formen der Kontextselektion für die Interpretation einhergehen: Faktische oder kontrafaktische Elemente lassen sich - wie die Begriffe ja bereits anzeigen stets auf jene Fakten der realen Welt beziehen, die sie reproduzieren respektive variieren, wohingegen realistische und fantastische Elemente keine solche Korrelierung mit Einzelinformationen der realen Welt erfordern oder erlauben.

Als bedeutende - wenn auch keineswegs exklusive - Funktionsbestimmung der Kontrafaktik wurde in der vorliegenden Studie das Politische angenommen. Zum ersten Mal wurde diese für das kontrafaktische Denken zentrale Funktion theoretisch umfassend erläutert und in einer Reihe von Textdeutungen herausgearbeitet. Bezogen auf die basalen psychologischen Mechanismen kontrafaktischen Denkens - das Aufstellen normativer Kontrastszenarien und die Identifikation kausaler Einflussfaktoren - sowie mit Blick auf den Fiktionsstatus kontrafaktischer Texte konnte eine Begründung dafür geliefert werden, weshalb sich die Kontrafaktik in besonderer Weise als Verfahren politischen Schreibens anbietet. Während die allgemeinen Realitätsabweichungen der Fantastik mitunter den Vorwurf des Eskapismus - also der Flucht aus der realen Welt in eine angenehme Traum- und Scheinwelt - provozieren, ist für die Kontrafaktik selbst noch in der Faktenvariation eine enge Bindung an die realweltlichen Fakten konstitutiv. Indem sie sich (implizit) auf Weltwissen bezieht, dieses Weltwissen aber zugleich auf signifikante Weise bearbeitet, entzieht sich die Kontrafaktik gleichermaßen dem Vorwurf des Eskapismus wie dem Vorwurf eines ,bloßen“ Realismus, also der, wie Moritz Baßler schreibt, „basale[n] Frage jeder realistischen Kunst, worin eigentlich noch ihr Kunstcharakter besteht, ihr poetischer Mehrwert, der sie von nicht-künstlerischen, etwa historischen, wissenschaftlichen oder eben journalistisch-dokumentarischen Darstellungen unterscheidet.“1326 Die konstitutiv dialektische Faktenbindung der Kontrafaktik - verstanden als par-

1326 Baßler: Realismus - Serialität - Fantastik, S. 37. Angesichts dieser in der Tat naheliegenden Frage überrascht es nicht, dass die allermeisten theoretischen Bestimmungen des Realismus - sei es vonseiten der Autoren fiktionaler Texte, sei es vonseiten der Literaturwissenschaft - bemüht sind, den Eindruck zu zerstreuen, es handele sich bei der realistischen Kunst um eine bloße Verdoppelung oder Abbildung der Realität. 
tielle künstlerische Autonomisierung gegenüber der realen Welt bei gleichzeitiger Bindung an selbige - eröffnet für kontrafaktische Texte vielfältige Möglichkeiten der Formulierung eines genuin künstlerischen, kritisch-politischen Gegenwartskommentars. Die primäre Funktion der Kontrafaktik ist dabei nicht so sehr epistemischer oder ontologischer Natur, sondern eher im Bereich des Emotiven, Normativen und Politischen verortet. Die, wenn man so will, funktionale Leitfrage der Kontrafaktik lautet nicht „Was wäre, wenn ...?“, sondern vielmehr „Wie wäre es, wenn ...“; wie also könnte oder müsste eine bestimmte, signifikant veränderte Variante der Realität bewertet werden.

In der vorliegenden Arbeit wurde erstmals die Kontrafaktik speziell in der deutschsprachigen Gegenwartsliteratur in den Blick genommen. Anhand umfassender Interpretationen der Romane Ich werde hier sein im Sonnenschein und im Schatten und Imperium von Christian Kracht, der Prosaarbeiten wir schlafen nicht und die alarmbereiten von Kathrin Röggla sowie der utopisch/dystopischen Romane Corpus Delicti von Juli Zeh und Schimmernder Dunst über CobyCounty von Leif Randt wurde die Prävalenz und formale Bandbreite kontrafaktischer Erzählverfahren in der deutschsprachigen Gegenwartsliteratur exemplarisch aufgezeigt. An zentrale Überlegungen des Theorieteils schließt der Interpretationsteil der Arbeit dabei unter anderem dadurch an, dass mit den Werken von Röggla, Zeh und Randt zugleich zwei Genres diskutiert wurden, deren Affinität zur Kontrafaktik in der bisherigen Forschung nicht bemerkt oder zumindest nicht hinreichend theoretisch begründet wurde: nämlich der kreative Dokumentarismus und die Utopie/Dystopie. Im Rahmen der Ausführungen zur (nicht-)realistischen Literatur seit 1945 und der konkreten Einzelanalysen konnte darüber hinaus die These von der Wahlverwandtschaft zwischen Kontrafaktik und politischem Schreiben literarhistorisch unterfüttert sowie werkanalytisch-exemplarisch belegt werden. Innerhalb der so überaus populären amimetischen Kunstformen der Gegenwart bildet gerade die Kontrafaktik ein privilegiertes künstlerisches Verfahren politischen Schreibens.

Auf der Basis des Vorschlags einer praxeologischen Modellierung des politischen Schreibens im zweiten Teil der Arbeit konnte schließlich im Nachgang der Interpretationen der Versuch eines politischen Vergleichs zwischen den literarisch-politischen Praktiken bei Christian Kracht, Kathrin Röggla, Juli Zeh und Leif Randt unternommen werden, Praktiken, die sich durch ein je spezifisches Zusammenspiel von Werkästhetik, Autorpoetik, Autorinszenierung und öffentlicher Rezeption auszeichnen. Die vier Autoren und ihre Werke wurden dabei auf tentative Weise den literarisch-politischen Praktiken der Provokation (Kracht), der Subversion (Röggla), des Engagements (Zeh) und der Affirmation (Randt) zugeordnet. Dabei konnte - bei aller Unterschiedlichkeit der vorgestellten literarisch-politischen Praktiken - nachgewiesen werden, dass 
den Realitätsvariationen der Kontrafaktik jeweils zentrale Bedeutung für das politische Schreiben von Kracht, Röggla, Zeh und Randt zukommt.

Während die literarhistorischen und interpretatorischen Ergebnisse der vorliegenden Arbeit als eigenständige und relativ abgeschlossene Forschungsergebnisse Gültigkeit beanspruchen können, laden die fiktions- und interpretationstheoretischen Überlegungen zum Weiterdenken ein. Von den in dieser Arbeit behandelten Fragen ausgehend sollen zum Ende hin einige Forschungsfelder benannt werden, in denen sich die neu eingeführte Kategorie der Kontrafaktik künftig als produktiv erweisen mag.

Historisierung: Zahlreiche Möglichkeiten der Anschlussforschung ergeben sich im Bereich einer Historisierung der Kontrafaktik. Für den deutschsprachigen Kulturraum ist noch nicht einmal das alternativgeschichtliche Erzählen, das den bisherigen Fokus der Forschung zum fiktionalen kontrafaktischen Erzählen gebildet hat, literarhistorisch befriedigend aufgearbeitet. Gibt es etwa in Deutschland, so könnte man fragen, ähnlich wie in Frankreich bereits im 19. Jahrhundert Beispiele fiktional breit ausformulierter Alternativgeschichte? In welchen historischen Situationen und unter welchen ideologischen Einflüssen entstehen die frühen Werke der deutschsprachigen historischen Kontrafaktik? Die Fragen der Historisierung potenzieren sich noch, wenn man weitere kontrafaktische Genres wie Dystopie, Satire oder Schlüsselroman miteinbezieht. Zwar liegt zu den einzelnen Genres bereits eine mehr oder weniger umfangreiche Spezialforschung vor. Der theoretische Zugriff der vorliegenden Studie erlaubt es jedoch, vorderhand von wohldefinierten Einzelgenres abzusehen und stattdessen eine integrative oder komparative Geschichte fiktionaler Realitätsvariationen in ganz unterschiedlichen Genres und Medien zu schreiben. Die Geschichte ,der‘ Kontrafaktik wäre dann nicht länger als die Geschichte eines bestimmten Genres zu konzeptualisieren, sondern würde vielmehr Teil einer integrativen Verfahrensgeschichte der Literatur bilden.

Geschichte der Fiktionalität: Während faktuales kontrafaktisches Denken zumindest als Form des Alltagsdenkens - wohl in den meisten, wenn nicht gar in allen menschlichen Kulturen vorkommt, können Werke der Kontrafaktik, also des fiktionalen kontrafaktischen Erzählens, nur dann verfasst und verstanden werden, wenn die entsprechende Kultur die ,Institution' Fiktionalität überhaupt ausgebildet hat. Daraus folgt, dass eine Historisierung der Kontrafaktik letztlich nur unter Berücksichtigung der Geschichte der Fiktionalität zu leisten ist. ${ }^{1327}$ Es stellt sich in diesem Zusammenhang etwa die Frage, ob und unter welchen Bedingungen man für die Literatur der Antike oder des Mittelalters von

1327 Vgl. Johannes Franzen u. a. (Hg.): Geschichte der Fiktionalität. Diachrone Perspektiven auf ein kulturelles Konzept. Baden-Baden 2018. 
Werken der Kontrafaktik sprechen kann. Ferner wäre zu untersuchen, ab welchem Zeitpunkt in der Literatur- und Geistesgeschichte und unter welchen kulturellen Bedingungen Formen der fiktionalen Kontrafaktik und der faktualen Kontrafaktizität auseinanderzutreten beginnen.

Publikumsspezifizierung: Bei der Kontrafaktik handelt es sich um ein kontextsensitives Erzählphänomen, das nur in Relation zu einer spezifischen (Modell-) Leserschaft und deren spezifischem Wissenshorizont expliziert werden kann. Wie in der Hermeneutik üblich, wurde im Rahmen der vorliegenden Studie von plausiblen Modell-Rezeptionssituationen ausgegangen. Als Modell-Leser wurde ein durchschnittlich informierter Text-Rezipient zur Zeit der jeweiligen Textveröffentlichung angesetzt; die Auszeichnung einzelner Textelemente als kontrafaktisch - oder auch als faktisch, realistisch oder fantastisch - wurde jeweils in Bezug auf das Weltwissen eines solchen Lesers vorgenommen.

So plausibel und gewissermaßen unausweichlich ein solches Vorgehen für die Literaturwissenschaft auch sein mag: Es handelt sich hier letztlich um eine idealtypische, überaus voraussetzungsreiche Konstruktion, welche die vielfältigen realen Leseerfahrungen nur annäherungsweise zu modellieren vermag und sie im Einzelfall mitunter gänzlich verfehlen wird. Unterschiedliche Rezipienten oder Rezipientengruppen verfügen über höchst unterschiedliche Ausformungen von Weltwissen, wobei die jeweiligen Einzelenzyklopädien durchaus in Konflikt zueinander stehen können. Ein einheitliches Bild der Realität, das allgemein akzeptiert wäre und mithin eine verlässliche Basis für fiktionale Faktenreferenzen und Faktenvariationen bereitstellen könnte, gibt es nicht. Die individuelle oder zumindest gruppenspezifische Konfiguration von Realitätsannahmen stellt nun für Fantastik und Realistik kein allzu großes Problem dar, da Grundüberzeugungen hinsichtlich des Wesens der Realität nur selten in Zweifel gezogen werden (wenngleich sich wohl kaum eine allgemeine Realitätsannahme finden lässt, die nicht sehr wohl einmal irgendwann von irgendjemandem in Zweifel gezogen worden wäre ${ }^{1328}$ ). Bei Faktik und Kontrafaktik hingegen - im Falle also einer Referenz auf konkrete Einzelfakten der realen Welt - gestaltet sich die Situation unvergleichlich schwieriger. Nicht nur lassen sich Fakten und Faktenvariationen einzig innerhalb bestimmter kultureller, sprachlicher und historischer Parameter

1328 So betont Monika Schmitz-Emans im Rahmen ihrer Diskussion der Fantastik, dass „unter den Mitgliedern einer Kommunikationsgemeinschaft kaum je ein Konsens über das ,Realitätskompatible‘ [besteht]. Für den, der an höhere Mächte glaubt, ist anderes ,realitätskompatibel` als für den Ungläubigen; für den einen sind Wunder eben doch ein Bestandteil des Weltbildes, für den anderen nicht. Und für den wissenschaftlich Ausgebildeten stellt sich die Welt oft ganz anders dar als für den Laien.“ (Monika Schmitz-Emans: Phantastische Literatur: Ein denkwürdiger Problemfall. In: Neohelicon XXII/2 (1995), S. 53-116, hier S. 65 f.). 
als mehr oder weniger allgemein gültig voraussetzen. Politisch brisante Phänomene wie Post-Truth, sogenannte ,alternative Fakten', Postfaktizität und Fake News haben in den letzten Jahren auf beunruhigende Weise deutlich gemacht, dass selbst innerhalb eines relativ homogenen Kulturraums keineswegs Einigkeit darüber besteht, welche Fakten allgemeine Gültigkeit beanspruchen können und anhand welcher Mechanismen einer bestimmten Annahme überhaupt Faktenstatus zugeschrieben werden kann. Holocaustleugner und Verschwörungstheoretiker mögen zwar nicht die intendierte Leserschaft der in dieser Studie analysierten Werke - noch auch dieser Studie selbst - sein. Im Rahmen einer Kartografierung des politischen Feldes der Gegenwart - inklusive seiner offenkundigen Verwerfungen - sind allerdings durchaus die Fragen von Bedeutung, welche fiktionalen Werke von welchen Teilpublika überhaupt als kontrafaktische Werke rezipiert werden (können), ob, wann und von wem konventionell als kontrafaktisch angesehene Werke als realistische, faktische oder fantastische Werke (miss-) gedeutet werden und welche gemeinhin nicht als kontrafaktisch klassifizierten Werke innerhalb spezifischer, gewissermaßen wahrheitsexzentrischer Rezeptionsgemeinschaften als Werke der Kontrafaktik aufgefasst werden. (Wie würde beispielsweise ein Holocaustleugner Harris' Fatherland deuten? Wo beginnt für den Kreationisten die Fantastik?)

Medialität: In der vorliegenden Arbeit wurde der Fokus auf Ausprägungen der Kontrafaktik in der fiktionalen Prosa gelegt. Medial ist die Kontrafaktik allerdings keineswegs an die fiktionale Prosa gebunden, sondern kann in allen fiktionalen Medien zum Einsatz kommen, also etwa auch im Film, im Comic oder im Drama. Im Gegensatz zur fiktionalen Prosa, welche ein Kommunikationssystem mit nur einem einzigen Code - gedrucktem Text respektive sprachlichen Zeiten - bildet, werden in anderen medialen Formaten verschiedene Codes miteinander kombiniert, also etwa Text, Bild, Musik etc. Durch diese multimediale Anlage verändern sich zugleich die Möglichkeiten einer (indirekten) Referenzialisierung realweltlichen Faktenmaterials - und damit auch die Möglichkeiten der Erzeugung kontrafaktischer Referenzstrukturen. So wäre es von großem Interesse, im Rahmen der Interpretation kontrafaktischer Comics wie etwa Alan Moores und Dave Gibbons' Watchmen oder Neil Gaimans und Andy Kuberts Marvel 1602 zu untersuchen, wie hier verschiedene mediale und semiotische Systeme zusammenwirken - oder auch einander entgegenarbeiten -, um kontrafaktische Referenzen zu produzieren. Im Falle kontrafaktischer Filme könnte man demgegenüber die Frage stellen, auf welche Weise die Einbeziehung des tendenziell nicht-referenziellen Mediums der Musik in Kombination mit anderen Medien zur Verstärkung oder Abschwächung, vielleicht sogar zur primären Generierung kontrafaktischer Referenzen beitragen kann. Schließlich lassen sich die Überlegungen zu einer formalen Kontrafaktik, welche im 
Rahmen dieser Studie vor allem in Bezug auf die Sprachvariationen im Werk Kathrin Rögglas und andeutungsweise in Bezug auf die Illustrationen ihrer Werke angestellt wurden, im Bereich der Bild- und Tonmedien respektive im Feld der Intermedialitätsforschung auf vielfältige Weise fortentwickeln.

Performativität: Weitreichende performativitätstheoretische und medienphilosophische Implikationen ergeben sich aus der Theorie der Kontrafaktik für Kunstformen, in denen fiktionale Repräsentationen durch die Realpräsenz von Objekten oder Personen ersetzt werden oder zumindest mit diesen parallellaufen: etwa bei der Einbeziehung von Readymades in die Aufführung fiktionaler Dramen, bei Cameo-Auftritten realer Personen in Film und Drama oder auch ganz allgemein bei der Darstellung fiktiver Figuren durch reale Schauspieler. Es stellt sich hier unter anderem die Frage, in welchem Sinne und unter welchen Bedingungen eine reale Entität in eine fiktionale Entität (teil-)transformiert werden kann und welchen Einfluss diese Transformation auf die Interpretation des jeweiligen Werkes hat. In welchem fiktions- und performativitätstheoretischen Verhältnis stehen etwa bei einer Theateraufführung die realen Schauspieler zu den fiktiven Figuren der freien Diegese ${ }^{1329}$ Wäre der reale Bundespräsident, der in einem Stück über den Bundespräsidenten auftritt, ein kontrafaktischer Bundespräsident? Kann der reale Körper eines Schauspielers, wenn er im Rahmen der Aufführung eines fiktionalen Stückes zum Zeichen wird, als kontrafaktische Version seiner selbst fungieren? Ist die Schauspielerin, welche Emilia Galotti spielt, während der Vorstellung in gewissem Sinne nicht immer zugleich eine kontrafaktisch-autofiktionale Version dieser Schauspielerin selbst?

Derartige Überlegungen gehen freilich weit über das Untersuchungsinteresse der vorliegenden Studie hinaus. Ihre Klärung muss nachfolgenden Arbeiten überlassen werden, denen die obigen Ausführungen vielleicht als Fingerzeig dienen können.

Entgegen der Ankündigung vom Beginn des Fazits, dass sich die Theorie der Kontrafaktik nicht anhand eines einzelnen literarischen Beispiels sinnvoll illustrieren lässt, sei abschließend doch noch einmal der Literatur selbst das Wort erteilt - allerdings nicht mit einem Beispiel der Kontrafaktik, sondern mit einer metafaktischen Theoriereflexion innerhalb eines kontrafaktischen Romans. In Ward Moores Alternate History-Klassiker Bring the Jubilee von 1953 unterhalten sich zwei Personen über den Nutzen der literarischen Fiktion:

1329 Zur Unterscheidung von Bühnendiegese und freier Diegese siehe Alexander Weber: Episierung im Drama. Ein Beitrag zur transgenerischen Narratologie. Berlin / Boston 2017, S. 171. Sinnvoll ist diese Unterscheidung freilich nur für Dramenformen, die überhaupt eine fiktionale Welt entwerfen - nur eingeschränkt also für die Performances des postdramatischen Theaters. 


\begin{abstract}
„Aber du bist kein Freund von Romanen, nicht wahr? [...] Vielleicht unterschätzt du den Wert der Erfindung.“

„Nein“, sagte ich, „aber welchen Wert hat die Erfindung von Ereignissen, die niemals stattgefunden haben, oder von Charakteren, die nie existierten?“

„Wer kann urteilen, was geschehen und was nicht geschehen ist? Es ist eine Frage der Definition.“

„Gut“, erwiderte ich. „Nehmen wir an, die Charaktere existieren im Geist des Autors, wie die Ereignisse; wo kommt der Wert der Erfindung ins Spiel?“

„In ihrem Zweck oder dem Gebrauch, der von ihr gemacht wird“, antwortete er. „Ein Rad, das sich ziellos in der Luft dreht, ohne etwas zu bewirken, ist nichts wert; dasselbe Rad an einem Wagen oder einem Flaschenzug kann schicksalsverändernd wirken.“

„Trotzdem kann man aus Märchengeschichten nichts lernen“, beharrte ich.

Er lächelte. „Vielleicht hast du nicht die richtigen Märchengeschichten gelesen.“1330
\end{abstract}

In seinem Nachdruck auf der Bedeutung des Tatsächlichen (,Ereignisse [...], die [...] stattgefunden haben“), der Frage nach der epistemischen Funktion der Erfindung (dem, was man „lernen“ kann) sowie seinem Generalzweifel an Wert und Funktion amimetischer Literatur („Erfindung“ und „Märchengeschichten“) nimmt der autodiegetische Erzähler hier genau jene fiktionsskeptische Haltung ein, die man in der Diskussion des (fiktionalen) kontrafaktischen Erzählens bis heute häufig findet. Sein Kombattant, der das Erfundene („was geschehen und was nicht geschehen ist“) zu einer „Frage der Definition“ erklärt, das funktionale Leistungspotenzial der Fiktion betont (,Zweck [und] Gebrauch“) sowie „den Wert der Erfindung“ verteidigt, kann hingegen als Vertreter jener Haltung zur Kontrafaktik angesehen werden, die auch in der vorliegenden Studie eingenommen wurde.

Die Beantwortung der Frage, welches nun die „richtigen Märchengeschichten“ sind, wird letztlich davon abhängen, was man von „Märchengeschichten“ oder allgemeiner: von der amimetischen Literatur - erwartet. Der metaphorische „Wagen“, vor den das „Rad“ der Kontrafaktik in dieser Studie gespannt wurde, war derjenige des Politischen. Freilich ist diese Funktionsbestimmung nicht exkludierend gemeint. Als Kunst verstanden, geht die Kontrafaktik auch im Politischen nicht auf. Gleichwohl ist die Kontrafaktik, wie gezeigt wurde, in besonderer Weise zum Verfahren politischen Schreibens prädestiniert. Die Kontrafaktik dreht sich, um im Bildbereich des obigen Zitats zu bleiben, nicht eitel um sich selber, sondern bildet ein dialektisch spannungsreiches Erzählverfahren, das sich zwar auf Fakten der realen Welt bezieht, im Umgang mit diesen Fakten aber die eigenen künstlerischen Lizenzen zur sachlichen und normativen Neuakzentuierung, Erfindung und Variation behauptet. Genau diese Spannung zwischen Faktenbindung und Realitätsdementi ermöglicht es der Kontrafaktik, als genuin künstlerisches Ver-

1330 Ward Moore: Der große Süden. München 2001, S. 86f. 
fahren der Gegenwartskritik produktiv zu werden. Alternativen zum Status quo oder besser: Variationen desselben - entwerfen kontrafaktische Werke dabei nicht so sehr, um zu zeigen, dass die Welt auch anders sein könnte; dies wäre letztlich eine ontologische Aussage, die der Kunst nicht ohne Weiteres zukommt oder abverlangt werden kann. Ihr spezifisches, ästhetisch-normatives Potenzial entfalten die politischen Realitätsvariationen der Kontrafaktik, indem sie die Welt in ihrem So-Sein kritisch reflektieren. 